\title{
Intravascular Volume Assessment What Is New?
}

A.M.Abd el-Hamid, M.A.Khashaba and S.M.Twfik

Anesthesiology and Intensive Care, Dept., Faculty of Medicine, Benha Univ., Benha, Egypt

E-mail:Twfik@yahoo.com

\begin{abstract}
Static and dynamic fluid reactivity indicators are used to guide the reactivation of fluid. Inadequate intravascular volume causes hemodynamic instability and organ dysfunction. Therefore, techniques for evaluating intravascular volume are extremely essential to save patient life.
\end{abstract}

Key words: Body fluids, Fluid volume status, Volume assessment, CVP, US, COVID 19.

\section{Introduction}

In perioperative and critical care, two principles are important to fluid status evaluations. Euvolemia indicates a normal amount of bodily fluid that allows for sufficient cardiac chambers to be filled and in turn enables the heart to generate a cardiac output that can respond to the body's need for oxygen. No diuresis or fluid administration is required in the context of euvolemia. Fluid response defines the heart's capacity to react to volume changes by changing the stroke volume and thus the cardiac output [1].

Hypervolemia and hypovolemia both may have negative effects on the function of the organ: hypervolemia by generating edoema and hypovolemia by restricting the supply of oxygen to the organs [2].

It is essential to identify the patient's hypovolemia and to benefit from fluid delivery. Given additional intravenous fluid, tissue hypoperfusion, such as arterial hypotension, oliguria, changed skin perfusion, or altered mentation should only be tried. [3] Hypervolemia has significant clinical implications if a patient is hypervolemic, Hypervolemia should be evaluated if a positive response to fluid is maintained and the arterial pressure is increased, while a positive urine response to a fluid challenge promotes the need to provide additional fluid [4].

\section{Body Fluids}

The human body is mostly water: the entire volume in two main compartments is distributed. The bigger part is in cells (intracellular fluid, ICF), the smaller the amount being extracellular (ECF). [5] the ECS is split into three more compartments: IVS, plasma, interstitial (ISS) and transcellular (TCS). These bodily water compartments are bordered by a semipermeable membrane, through which liquids move from one place to another and divide them. [6] Osmolarity measure solution concentration as the number of solute particles per $1 \mathrm{~L}$ solution. Osmolarity per litre of solution is measured in milliosmoles $(\mathrm{mOsm} / \mathrm{L})$. It is computed as a product of the molarity and of the Van't Hoff coefficient that takes into account the degree of dissociation of the solution present. For example, when a solution includes 1 glucose mole or 1 $\mathrm{NaCl}$ mole it is 1 osmolar in glucose (which doesn't distinguish in solution) whereas it is 2 osmolar in $\mathrm{NaCl}$ (which dissociates in solution into sodium ions and chloride ions) [7]

Osmolality is another measure of the solution concentration given as the number of particles per 1 kilogramme of solution water. The osmolality is measured per kilogramme $(\mathrm{mOsm} / \mathrm{kg})$ of water in milliosmoles. Although osmolarity and osmolarity are often linked, their significance is somewhat different; osmolarity, in reality, represents a solution of osmotic concentration per volume of solution while osmolarity is by mass of solvent. The osmolality of the plasma is mainly controlled by $\mathrm{ADH}$, a hormone generated by the hypophysis in response to an increase in plasma osmolality (closely determined by the plasma sodium concentration). $\mathrm{ADH}$ determines an increase in the reabsorption of water from the kidney and thus the correction of the elevated osmolality. [8]

Another factor that controls fluid flow across biological membranes is oncotic pressure or colloidosmotic pressure e. Water will thus go from low to high-protein solutions. [9]

The selection of a plasma non-blood replacement should be guided by the characteristics of the desired fluid space. Ideal IV fluids should not induce electrolyte imbalances and should include metabolic anions. [10]

A hypotonic solution lowers the plasma osmotic pressure that leads to water transfer from the ECS to the ICS. [11]

Cellular edoema and lysis may happen Larger quantities of hypotonic solutions were known to lead to a temporary rise in ICP [12]

Many clinical environments may raise osmotic plasma pressure with a very high death rate. Mortality is clearly linked with plasma osmolality in both hyperosmolar non-ketotic syndrome and diabetic ketoacidosis. [13]

The tonicity of two solutions, separated by a semi-permeable barrier, is a comparative measure of osmotic pressure. Under this situation, water moves from a less osmotic solution (hypotonic solution with a low number of solution particles) to a solution with greater osmotic activity (hypertonic solution with higher number of solute particles). Tonicity is characterised as the osmotic pressure differential between the two liquids. [14] 


\section{Hypovolemia}

Hypovolemia is a low intravascular volume of the blood, with low average systemic filling pressures and a net flow of water and electrolytes into the bloodstream from the interstitial and tissue spaces. This supports blood volume, but reduces tissue stiffness and vascular reserve loss. [15] The most frequent cause of hypovolemia is bleeding. Indeed, direct blood loss may very rapidly lead to hypovolemia. The site of bleeding may be internal (such as bleeding into the abdomen), gastrointestinal (stomach haemorrhage, oesophagus or bowel bleeding) or exterior. Sometimes the signs and symptoms of hypovolemia are the initial indicators for internal or gastrointestinal haemorrhage rather than the observation of the fluke itself. [16] Severe hypovolemia and reduced peripheral perfusion is seen in patients with hypovolemic shock. Untreated, these individuals may suffer ischemia damage to critical organs leading to organ insufficiency in many systems. The first issue is whether the hypovolemic shock is caused by bleeding or fluid losses, as therapy will be dictated. When the cause of the hypovolemic shock is identified, blood or fluid loss must be replaced in order to reduce tissue ischemia as quickly as feasible. The pace of fluid replacement and the kind of fluid to be utilised must be considered when replacing fluid loss. [17] The symptoms may vary with the prior function level of the organ, compensatory mechanisms, organ failure and shock-like causes. The hypovolemic shock symptoms include pallors, tachycardia, hypotension, dyspnea, diaphoresis, tachyphoresis, cyanosis, weak heart sounds, agitation, changes in mental condition, pupils pinpoint, cold, clamma, lactic acidosis and low urinal outputs. [18] understand the state of any ongoing bleeding or loss of volume, need for transfusion and surgery, and investigate the potential to contribute to non-hemorrhagic causes such as stress pneumothorax and pericardial tamponade. Fluid response is arbitrarily defined as a $15 \%$ or higher increase in cardiac output to meet a fluid problem and patients are thus classified into 'responder and non-responder' The clinician attempts to maintain oxygen supply to tissues until the bleeding is stopped, minimising tissue hypoxia, inflammation, and organ failure. [19] The use of fluid resuscitation, vasopressors, and blood transfusion are part of this endeavour. Currently the expansion of volume attempts to enhance global blood flow in the expectation that this would increase the flow of microcirculations to increase the availability of oxygen in tissues. [19] whether colloids or crystalloids should be employed, and more precisely which colloids. Questions that continue to be addressed include the choice of resuscitation fluid, the aim of hemodynamic targets for bleeding management and optimum prevention of traumatic coagulopathy. [20] As crystalloid resuscitation requires more volume, it is more likely to cause tissue edoema and abdominal compartment syndrome. Excessive tissue hydration was attributed for greater incidence after abdominal operation of post-operative nausea and anastomotic dehiscence [20].

\section{Hypervolemia}

Hypervolemia refers to an excessive blood volume, wherein mean systemic circulatory pressure is high, causing a net fluid loss into the interstitium resulting in some edema formation. [21]

Hypervolemia is likely to be either iatrogenic or the result of oliguric renal failure or heart failure. In the case of oliguric renal failure, it is difficult to reduce the blood volume without dialysis [22].

The primary risk of hypervolemia is related to hypertension and an increase in myocardial work, which could lead to failure in a heart with marginal reserve. Hypervolemia also may lead to pulmonary edema. [22]

Hypervolemia also may lead to pulmonary edema. Hypervolemia must be aggressively managed with IV diuresis, including infusions of loop diuretics, furosemide (Lasix 40 to $100 \mathrm{mg} / \mathrm{hr}$ ), or bumetanide (Bumex 1 to $2 \mathrm{mg} / \mathrm{hr}$ ), or by continuous renal replacement therapy (CRRT), which should be initiated if the urine output on diuretic infusion is inadequate or if renal failure is present. [23]

\section{Intravascular Fluid Assessment Methods}

The clinical examination may anticipate extremes of body water. Tachycardia, hypotenesis and skin turgor loss in non-anesthetic adults are linked with open hypovolemia whereas skin and sunken eyes and fontainous tensions may be seen in babies. The detection of hypervolemia depends primarily on cardiac pathology, with lesser grades of fluid excess, such as rales, 3rd heart sound, oxygen deaturation, distension of the jawbone vein and peripheral edoema measurement of blood pressure response. The examination of the neck veins and the passive leg raising test may provide valuable information according on patient posture and access. The passive leg elevation test (PLR) presents a reversible endogenous fluid challenge via an increased venous return, which in a supine patient raises the legs to 45 degrees and evaluates the effects of it on blood pressure and heart rate. One easy method to accomplish PLR is to move a 45 degree recumbrent patient horizontally with a supine trunk and raised legs utilising the pivotal movement of the bed. [24] Central Venous pressure (CVP) is the thoracic pressure measurement in the superior vena cava (SVC)) and is a suitable substitute for the equivalent right atrial pressures. CVP is the most frequently utilised intravascular volume measurement method. [25] Catheter of the pulmonary artery (PACs) and pulmonary artery Pressures Occlusion (Wedge). Right heart conformity aberrations and pulmonary vascular resistance as a result of heart and lung disease may significantly change the connection between CVP and left heart precharge. Therefore, pulmonary artery catheterization remained an appealing choice in 
critically sick patients with such underlying disease for both right and left heart and pulmonary arterial hypertension. [26] The common intravascular fluid assessment technique Transthoracic Echo ( $T$ T E) provides fast, non-invasive, fluid and functional evaluation. Direct measurements of the lower venum cava diameter (IVC) changes with breathing and also right and left ventricular end diastolic volumes may be obtained with reliable cardiac preload. A reduction of 50 percent in IVC diameter (caval indice), seen in spontaneous breathing subcostal vision associated with a RA pressure of less than $10 \mathrm{~mm} \mathrm{Hg}$ (mean SD $6 \pm 5$ ), assessed in CVP In mechanically ventilated patients, Delta DIVC distinguished individuals with IVC variations of 12 percent and responding with higher $\mathrm{CO}$ to an unresponding fluid bolus. [27]

Cardiography of the transesophageal echo The primary emphasis for general usage is the intravascular status evaluation and the heart function in general. In ICU, TEE is indicated when TTE cannot properly acquire diagnostic information. [28] As with TTE for the IVC preload assessment, TEE may be utilised to measure SVC collapse. [29]

\section{Volume assessment in critically COVID19 patient}

Coronavirus 2019 (COVID-19), caused by new acute coronavirus 2 syndrome (SARS-CoV-2), is spreading fast all over the world. Whereas most infected individuals have a moderate condition, about 5 percent develop severe disease necessitating ICU hospitalisation, which is significant in absolute numbers. [30] The state of volumes of COVID-19 patients is dynamic, ranging from severe hypovolemia to open hypervolemia. A subgroup of patients with COVID-19 with anorexia, vomiting, and diarrhoea that need fluid resuscitation with hypovolemia. [31] On the other hand, ARDS and distributive shock may occur later in the course of the illness and continuous fluid expansion could result in harmful effects which exacerbate the anomalies of the pre-existing gas exchange, especially in the oliguric AKI. [32] individuals who died of COVID-19 showed right ventricular dilation cardiomegaly that may be due to ARDS itself being exacerbated by a fluid overload and a positive pressure ventilation impact. [33] Paradoxically, excessive efforts to keep such patients "dry" may not be an ideal approach, because a reduction in cardiac outputs and pulmonary blood flow would result in an increase in alveolar and physiological space which would eventually exacerbate the gas exchange. [34] Furthermore, a recognised characteristic of COVID-19 is the cytokine storm which may lead to greater vascular permeability, severe pulmonary edoema, fluid loss in the third space, intraabdominal hypertension, and multi-organ failure. [35] Conventional physical examination has limited diagnostic use in hemodynamically unstable patients to anticipate fluid and to guide vasoactive treatment. [36] Hypovolemia is not all hypotension. Interestingly enough, in individuals with COVID-19 unusual manifestations have been described, such as cardiac failure with acute myopericarditis, which may be linked with hypoension but not volume depletion. [37] Lung isolation or inferior vena cava (IVC) ultrasonography may be restricted. For instance, the results of lung ultrasound (LUS) in ARDS may be difficult to differentiate from the findings of cardiovascular pulmonary oedema, and patient posture can sometimes restrict the areas for assessment accessible. Similarly, because of its reliance on intraabdominal pressure and variations in tidal volume, local mechanic variables (e.g. thrombosis, IVC filter, cannula of the venous), and the patients' inspirational efforts, IVC ultrasonography may not be accurate. [38] The typical features of COVID-19 on LUS, while largely non-specific, are a thicker or uneven pleural line, subpleural consolidation and multifocal or Bconflict lines. [38] Patients with ARDS have similar characteristics, although more extensive and bilateral with B-line confluence, suggesting aeration loss and greater extravascular lung water. As previously stated, it may be hard to differentiate between this look and cardiogenic pulmonary edoema that can also be observed in COVID-19 individuals. However, some characteristics may assist to distinguish these two entities, reducing or not having pleural sliding and the existence of lung pulses and sparing regions are strongly evocative of ARDS, while pleural effects are observed more often in cardiogenic edoema. [39] Furthermore Lung US aeration scores may be utilised to evaluate treatment response and for prognosis in COVID-19 ARDS patients. [40] LUS is also a useful technique for detecting and guiding the proper intervention problems of positive pressure ventilation like as pneumothorax. [41]

\section{Summary}

Evaluating the volume of a patient is important if the patient in ICU is to be preoperative, hemodynamically unstable and severely sick. Insufficient intravascular volume may result in reduction in tissue oxygen and organ dysfunction whereas fluid excess, including respiratory failure, leads to edoema and organ dysfunction.

Measurement of intra-vascular volume, including clinical symptoms and signs, sound assessment of inferior diameters (IVC) of vena cava and IVC collapsibility index (IVC-CI), which seem valid markers both of intravascular volume status and of clinical volume recovery response. Although central venous (CVP) and pulmonary capillary wedges (PCWP) pressure measurement is one of those techniques for assessing the volume status, it cannot be consistently predicted.

\section{References}

[1] Sumit Singh, G.Ware Kuschner, Geoffrey Lighthall. Perioperative Intravascular Fluid Assessment andMonitoring: A Narrative Review of Established and Emerging Techniques. 
Anesthesiology Research and Practice, vol. Article ID 231493,pp. 11, 2011.

[2] J.L.Vincent: Fluid management in the critically ill. Kidney Int.vol.96,pp.52-57, 2019.

[3] A.Joosten, S.Raj Lawrence, A.Colesnicenco, S.Coeckelenbergh, J.L.Vincent, P.Van der Linden, M.Cannesson, J.Rinehart: Personalized versus protocolized fluid management using noninvasive hemodynamic monitoring (Clearsight System) in patients undergoing moderate-risk abdominal surgery. Anesth Analg.vol.1,pp.8-21, 2019.

[4] X.Monnet, J.L.Teboul: Passive leg raising: Five rules, not a drop of fluid! Crit Care.vol.2,pp.1938,2015 .

[5] A.Gillian Cockerill and A.Stephen, Reed Essential Fluid, Electrolyte and pH Homeostasis, First- Edition.vol.8,pp.78-90,2012.

[6] D.Chappell, M.Jacob, K.Hofmann-Kiefer, P.Conzen, M.Rehm, A rational approach to perioperative fluid management Anesthesiology .vol.109,pp.723-40,2008.

[7] E.B.Hendry and A.Clin Chem, Osmolarity of human serum and of chemical solutions of biological importance.vol.14,pp.36-61,1961.

[8] D.Voet, G.J.Voet, C.W.Pratt ,Fundamentals of biochemistry. Wiley, New York.vol.30 ,pp.350460,2001.

[9] M.A.Mansoor, B.J.Sandmann, Applied physical pharmacy. McGraw-Hill Professional.vol.1,pp.1525,2002.

[10] R.Zander,Infusion fluids: why should they be balanced solutions Eur Journ Hospital Pharmacy Practice.vol.12,pp.60-62,2006.

[11] E.L.Williams, K.L.Hildebrand, S.A.McCormick et al :The effect of intravenous lactated Ringer's solution versus $0.9 \%$ sodium chloride solution on serum osmolality in human volunteers. Anesth Analg .vol.88,pp.999$1003,1999$.

[12] C.Tommasino, S.Moore, M.M.Todd, Cerebral effects of isovolemic hemodilution with crystalloid or colloid solutions. Crit Care Med.vol.16,pp.862-868,1988.

[13] M.Jayashree, S.Singhi,Diabetic ketoacidosis: Predictors of outcome in a pediatric intensive care unit of a developing country. Pediatr Crit Care Med 5.vol.15,pp.427-433,2004.

[14] R.D.Miller, Miller's anesthesia, 7 edn. Churchill Livingstone, UK.vol.7,pp.150210,2009 .

[15] C.H.Shin, D.R.Long, D.McLean, S.D.Grabitz, K.Ladha, F.P.Timm, T.Thevathasan, A.Pieretti, C.Ferrone, A.Hoeft, T.W.L.Scheeren, B.T.Thompson, T.Kurth, M.Eikermann: Effects of Intraoperative Fluid Management on Postoperative Outcomes: A Hospital Registry Study.vol.267,pp.1084-1092,2018.

[16] J.Van der Mullen, R.Wise, G.Vermeulen, P.J.Moonen, Malbrain MLNG: Assessment of hypovolaemia in the critically ill. Anaesthesiol Intensive Ther.vol.50,pp.141-149,2018.

[17] N.Hooper, T.J.Armstrong, StatPearls [Internet]. StatPearls Publishing; Treasure Island (FL): Hemorrhagic Shock. [PubMed].vol.5,1551,2020 .

[18] L.I.Worthley, Shock: A review of pathophysiology and management. Part II. Crit Care Resusc.vol.2,pp.16-26,2000.

[19]

.Gruartmonera, J.Mesquidaa, C.Inceb, Fluid therapy and the hypovolemic microcirculation. [20] Curr Opin Crit Care.vol.9,pp.67-84,2015.

.Bouglé, A.Harrois, J.Duranteau, Resuscitative strategies in traumatic hemorrhagic shock. Ann Intensive Care.vol.1,pp.3-21,2013.

[21]

.K.Thacker, W.K.Mountford, F.R.Ernst, M.R.Krukas, M.M.Mythen,Perioperative Fluid Utilization Variability and Association With Outcomes: Considerations for Enhanced Recovery Efforts in Sample US Surgical Populations. Ann Surg.vol.3,pp.52-100,2016.

[22]

.Peter J. Pascoe, in Fluid, Electrolyte, and AcidBase Disorders in Small Animal Practice (Fourth Edition).vol.8,pp.81-98,2012.

[23]

.Greg, M.CKenna, B.Göran, G. Klintmalm, in Transplantation of the Liver (Third Edition).vol.3,pp.20-30,2015.

[24] J.Jabot, J. L. Teboul, C. Richard, and X. Monnet, "Passive leg raising for predicting fluid responsiveness: importance of the postural change," Intensive Care Medicine.vol.35,pp.8590, 2009.

[25] J. Boldt, M. Lenz, B. Kumle, and M. Papsdorf, "Volume replacement strategies on intensive care units: results from a postal survey," Intensive Care Medicine.vol.24,pp.147151,1998

[26] J. L. Vincent, "The measurement of right ventricular ejection fraction," Intensive Care World.vol.7,pp.333-336,1990.

[27] B. J. Kircher, R. B. Himelman, and N. B. Schiller, "Noninvasive estimation of right atrial pressure from the inspiratory collapse of the inferior vena cava," American Journal of Cardiology, vol. 66,pp.493-496,1990.

[28] D. M. Thys, Z. Hillel, M. E. Goldman, B. P. Mindich, and J. A. Kaplan, "A comparison of hemodynamic indices derived by invasive monitoring and two-dimensional echocardiography," Anesthesiology.vol.67,pp.630-634, 1987.

[29] R.Vieillard-Baron, S.Augarde, B.Prin, A.Page, B.Eauchet, and F. Jardin, "Influence of superior vena caval zone condition on cyclic changes in right ventricular outflow during 
respiratory

Anesthesiology.vol.95,pp.1083-1088,2001.

[30] Z. Wu, J.M. McGoogan, Characteristics of and important lessons from the coronavirus disease 2019 (COVID-19) outbreak in China: summary of a report of 72314 cases from the Chinese Center for Disease Control and Prevention. JAMA.vol.3,pp.12-39,2020

[31] J.Phua, L.Weng, L.Ling, M.Egi, C.M.Lim, J.V.Divatia, et al, Asian Critical Care Clinical Trials Group. Intensive care management of coronavirus disease 2019 (COVID-19): challenges and recommendations. Lancet Respir Med.vol.5,pp.56-71,2020.

[32] J.Bouchard, S.B.Soroko, G.M.Chertow, J.Himmelfarb, T.A.Ikizler, E.P.Paganini, et al.; Program to Improve Care in Acute Renal Disease (PICARD) Study Group. Fluid accumulation, survival and recovery of kidney function in critically ill patients with acute kidney injury. Kidney Int.vol.4,pp.42-72,2009.

[33] S.E.Fox, A.Akmatbekov, J.L.Harbert, G.Li, J.Quincy Brown, Vander Heide RS. Pulmonary and cardiac pathology in Covid-19: the first autopsy series from New Orleans. medRxiv.vol.1,pp.1-10,2020.

[34] J.I.Keddissi, H.A.Youness, K.R.Jones, G.T.Kinasewitz, Fluid management in acute respiratory distress syndrome: a narrative review. Can J Respir Ther.vol.5,pp.55-88.2018

[35] C.Ronco, T.Reis, Kidney involvement in COVID-19 and rationale for extracorporeal therapies. Nat Rev Nephrol.vol.4,pp.272-84,2020.

[36] B.Saugel, S.Ringmaier, K.Holzapfel, T.Schuster, V.Phillip, R.M.Schmid, et al. Physical examination, central venous pressure, and chest radiography for the prediction of transpulmonary thermodilution-derived hemodynamic parameters in critically ill patients: a prospective trial. J Crit Care.vol.2,pp.10-40,2011.

[37] R.M.Inciardi, L.Lupi, G.Zaccone, L.Italia, M.Raffo, D.Tomasoni, et al. Cardiac involvement in a patient with coronavirus disease (COVID19). JAMA Cardiol .vol.10.pp,10-19,2019.

[38] Q.Y.Peng, X.T.Wang, L.N.Zhang, Chinese Critical Care Ultrasound Study Group (CCUSG). Findings of lung ultrasonography of novel corona virus pneumonia during the epidemic.vol.46,pp.849-950,2020.

[39] R.Copetti, G.Soldati, P.Copetti, Chest sonography: a useful tool to differentiate acute cardiogenic pulmonary edema from acute respiratory distress syndrome. Cardiovasc Ultrasound.vol.1,pp.1-16,2008.

[40]

.T.Wang, X.Ding, H.M.Zhang, H.Chen, L.X.Su, D.W.Liu, Chinese Critical Ultrasound Study Group (CCUSG). Lung ultrasound can be used to predict the potential of prone positioning and assess prognosis in patients with acute respiratory distress syndrome. Crit Care.vol.5,pp.32-85,2016.

[41] M.Kennedy Hall, E.C.Coffey, M.Herbst, R.Liu, J.R.Pare, R.Andrew Taylor, et al. The "5Es" of emergency physicianperformed focused cardiac ultrasound: a protocol for rapid identification of effusion, ejection, equality, exit, and entrance.vol.5,pp.83-93,2015. 